\title{
Raman optical activity study of poly-L-proline chains of various lengths
}

\author{
V. Profant ${ }^{\mathrm{a}, *}$, M. Šafař́k ${ }^{\mathrm{b}}$, P. Bouř ${ }^{\mathrm{b}}$ and V. Baumruk ${ }^{\mathrm{a}}$ \\ ${ }^{a}$ Faculty of Mathematics and Physics, Institute of Physics, Charles University in Prague, Prague, \\ Czech Republic \\ ${ }^{\mathrm{b}}$ Institute of Organic Chemistry and Biochemistry, Academy of Sciences of the Czech Republic, Prague, \\ Czech Republic
}

\begin{abstract}
Raman and Raman optical activity (ROA) spectra of several oligo- and poly-L-proline samples of various chain lengths were measured in a wide frequency range between 120 and $1800 \mathrm{~cm}^{-1}$ and analysed with respect to the main peptide chain conformation. Specifically, formation of polyproline II (PPII) helical conformation was studied in dependence on the increasing chain length $N$ of the (L-proline) $)_{N}$ sample. Due to high sensitivity of the ROA technique to the conformational stability and rigidity of peptide chain we were able to determine the characteristic spectral peaks associated with formation of stable PPII helical conformation in studied systems. The most relevant peaks are located at 405,535 and $945 \mathrm{~cm}^{-1}$. Additionally, based on our data analysis, we were able to determine the minimal length of (L-proline) $)_{\mathrm{N}}$ chain necessary for creation of the stable PPII conformation as $N=6$.
\end{abstract}

Keywords: L-proline, Raman spectroscopy, Raman optical activity, polyproline II helical conformation, protein secondary structure

\section{Introduction}

Due to the high sensitivity of chiroptical methods to minor changes of the 3D structure of studied systems and possibility of study molecules in solutions, these procedures have become well established techniques in investigation of peptide and protein conformations. Comparing the three proven chiroptical spectroscopies: electronic circular dichroism (ECD) [6], vibrational circular dichroism (VCD) [9] and Raman optical activity (ROA) [3]; the application of ROA to the structural elucidation of proteins and peptides occurs to be the most recent [10]. Nevertheless, the basic set of secondary structure markers has already been specified [1].

Although a complete set of characteristics of certain secondary structures of proteins and peptides, such as several types of helical structures and beta sheets, is generally known, there are still conformations which remain unspecified. Specifically, there are peptide conformations responsible for so-called random-coil structure that are left uncharacterized. Recently, a number of evidence has lead to a presupposition that the random-coil structure is not just a statistically random mixture of conformations, but it is more organized and complicated [12].

\footnotetext{
*Corresponding author: V. Profant, Faculty of Mathematics and Physics, Institute of Physics, Charles University in Prague, Ke Karlovu 5, Prague 2 CZ-121 16, Czech Republic. Tel.: +420 221911 346; Fax: +420 224922 797; E-mail: profant@karlov.mff.cuni.cz.
} 
It is most probable that the main content of this structure is polyproline II helical conformation (PPII), which is left-handed and can be stabilized by solvent (spec. water) molecules; however, there are no internal stabilizing hydrogen bonds in it. It was confirmed that even short segments of PPII structure are surprisingly conformationally stable. Thus, there is a natural interest to study this structure. Actually, there are several ROA studies of non-proline peptides adopting PPII conformation [2]. However, just a few of them focused on polyproline itself [7].

In our experiment, the comparative analysis of oligo- and polyproline chains (including poly-L-proline as a linear standard of PPII conformation) was carried out using methods of Raman scattering and Raman optical activity in order to get better understanding of the formation and the occurrence of PPII conformation. For the analysis, five (L-proline) $)_{N}$ oligopeptides of different lengths $(N=2,3,4,6,12)$ and three commercial poly-L-proline samples of different mean molecular weight were used. Spectra were recorded in a wide frequency region between 120 and $1800 \mathrm{~cm}^{-1}$. The whole study was carried out as a continuation of our previous work [7], being extended and more thoroughly elaborated.

\section{Experimental section}

The poly-L-proline samples were purchased from Sigma-Aldrich, i.e. P-2254 (MW = 1000-10,000), P-2129 (MW = 10,000-30,000) and P-3886 (MW > 30,000). The oligopeptide (L-proline) $)_{6}$ and (L-proline) ${ }_{12}$ samples were acquired from IOCB ASCR (Institute of Organic Chemistry and Biochemistry of Academy of Sciences of the Czech Republic), where they were synthesized by Martin Šafařík; (L-proline $)_{2}$ (G-3060), (L-proline) $)_{3}$ (H-4795) and (L-proline) $)_{4}$ (H-4800) were purchased from Bachem.

Raman and Raman optical activity spectra were recorded on Prague ROA spectrometer [5]. The spectrometer employs the incident circular polarization (ICP) modulation scheme in backscattering geometry, which appeared to be most beneficial approach for biomolecular ROA. The spectrometer is based on a fast stigmatic spectrograph HoloSpec HS-f/1.4 (Kaiser Optical Systems) equipped with a holographic transmission grating and a back-illuminated CCD detection system (Roper Scientific, $1340 \times 100$ pixels).

The peptide solutions were prepared at nearly saturated concentration in doubly distilled deionized water at ambient temperature $(\sim 293 \mathrm{~K})$ and subsequently filtered through a $22 \mu \mathrm{m}$ Millipore filter into a standard $5 \times 5 \mathrm{~mm}^{2}$ quartz microfluorescence cell (Hellma). In order to minimize and stabilize spectral background, residual fluorescence coming from traces of impurities in the peptide was quenched by leaving the sample to equilibrate in the laser beam for several hours before the acquisition. All spectra were measured using $514.5 \mathrm{~nm}$ excitation laser wavelength and spectral resolution of $\sim 6.5 \mathrm{~cm}^{-1}$. Typical laser power at the sample was $500-600 \mathrm{~mW}$. The concentrations/acquisition times of particular samples were as follows: $0.5 \mathrm{M} / 13 \mathrm{~h}$ for (L-proline) $)_{2}, 0.3 \mathrm{M} / 18 \mathrm{~h}$ for (L-proline) $)_{3}, 0.3 \mathrm{M} / 25 \mathrm{~h}$ for (L-proline) $)_{4}$, $33 \mathrm{mM} / 76 \mathrm{~h}$ for (L-proline) $)_{6}, 17 \mathrm{mM} / 57 \mathrm{~h}$ for (L-proline) $)_{12}, 30 \mathrm{mg} \cdot \mathrm{ml}^{-1} / 53.5 \mathrm{~h}$ for poly-L-proline $\mathrm{P}-2254,20 \mathrm{mg} \cdot \mathrm{ml}^{-1} / 40.5 \mathrm{~h}$ for poly-L-proline P-2129 and $10 \mathrm{mg} \cdot \mathrm{ml}^{-1} / 62 \mathrm{~h}$ for poly-L-proline P-3886.

The solvent signal was subtracted from Raman spectra and then a baseline correction was made. A raw ROA spectrum was Fourier filtered to suppress quasi-periodic high spatial-frequency signal in the low wavenumber region, corrected for an artifact spurious signal from solvent and cell walls and finally subjected to a minor baseline correction when necessary [8]. Nevertheless, some artifacts caused by presence of tiny amount of impurities in sample (from the synthesis) may occasionally occur (and are marked by the asterisk in Fig. 1). All the data were treated using GRAMS/AI software (Thermo Electron Corporation). 

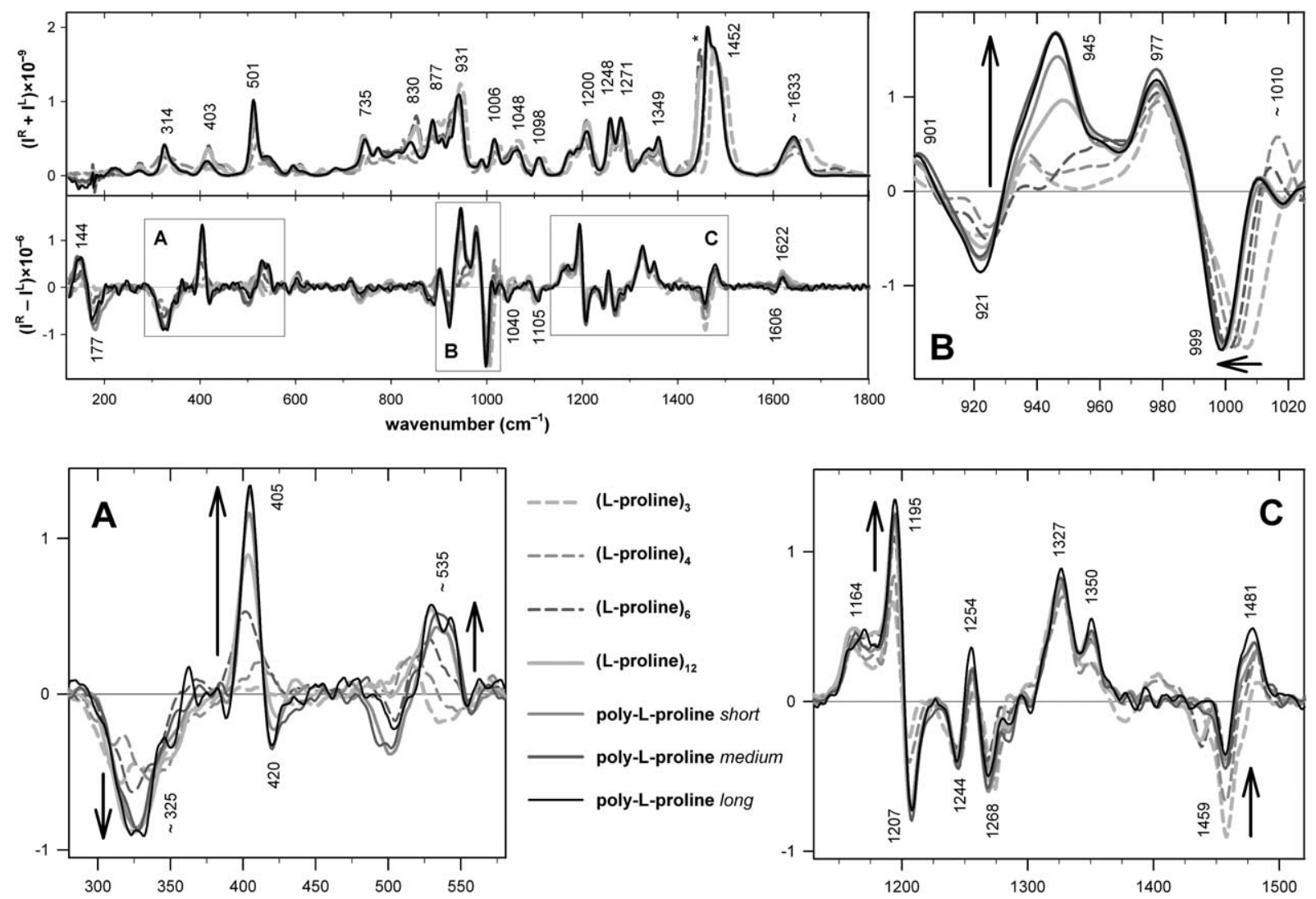

Fig. 1. Length dependence of the experimental Raman and ROA spectra of oligo- and poly-L-proline samples. Three polyprolines of different mean molecular weight were used: short (P-2254, MW $=1000-10,000)$, medium (P-2129, $\mathrm{MW}=10,000-30,000)$ and long $(\mathrm{P}-3886, \mathrm{MW}>30,000)$. Excisions A, B and C contain the enlarged parts of ROA spectral regions with major spectral bands evolvement. The black arrows indicate the major changes observed in ROA spectra that can be correlated with increasing chain length.

In order to compare spectra which were recorded under different experimental conditions, we normalized them on the same scale. Raman spectra were normalized on the spectral band at $1452 \mathrm{~cm}^{-1}$ and ROA spectra on the band at $\sim 1000 \mathrm{~cm}^{-1}$.

\section{Results and discussion}

Acquired back-scattered Raman and ROA spectra of studied proline samples are shown in Fig. 1. The spectra are plotted in the form of overlaid in order to emphasize the progression of the spectral bands with increasing chain length of prolines. Moreover, there are three enlarged excisions (A, B and C) from the ROA spectra displaying the spectral evolvement related to increasing chain length (intensity increase/decrease and band shifts) in closer details.

Presented spectra are plotted covering most of the frequency region available by our spectrometer within the limits of 120 and $1800 \mathrm{~cm}^{-1}$. It should be noted that our low wavenumber limit is due to recently upgraded instrument much lower than the usual $600 \mathrm{~cm}^{-1}$. However, the broad Raman signal 
of water bellow $200 \mathrm{~cm}^{-1}$ strongly interferes with the solute response in this region, which makes measurements bellow this limit less reliable.

Detection of PPII peptide conformation is usually based on the presence of well established ROA markers [11]: positive band at $1320 \mathrm{~cm}^{-1}$ in the extended amide III region and weak slightly asymmetric $(-+)$ couplet with broadened positive lobe in the amide I region at $1675 \mathrm{~cm}^{-1}$. However, due to the fact that the amide III mode does not occur in tertiary amides, the first marker is not present in oligo- and poly-L-proline ROA spectra and the second marker is substantially shifted $\left(1622 \mathrm{~cm}^{-1}\right)$. Nevertheless, there are also other characteristic band groups indicating presence of the PPII conformation in prolinecontaining peptides: well resolved ROA spectral patterns $(+-++-)$ in the $850-1050 \mathrm{~cm}^{-1}$ region and $(++-)$ in the $1100-1250 \mathrm{~cm}^{-1}$ region $[7,8]$. Both of these patterns are easily recognizable in Fig. 1.

Overlaid spectra in Fig. 1 illustrate an obvious development of ROA signal with increasing length of proline chain going from oligopeptide consisting of only 3 residues to polypeptide. Spectrum of proline dipeptide was omitted because its rather different shape is the crossover between a spectrum of single amino acid and longer oligopeptides.

Although most of spectral bands preserve an identical position and intensity independently on the peptide chain length, we can observe some interesting evolutions of intensities and even a shift of band position. There is apparent sharpening of the ROA spectra in the low wavenumber region (Fig. 1A) and gradual increase of three spectra bands: negative at $\sim 325 \mathrm{~cm}^{-1}$ and two positive at 405 and $\sim 535 \mathrm{~cm}^{-1}$. The most noticeable peak formation clearly related to increasing chain length is localized at $945 \mathrm{~cm}^{-1}$ (Fig. 1B). One can also observe slight increase of the positive ROA peak at $1195 \mathrm{~cm}^{-1}$ and decrease of the negative ROA peak at $1459 \mathrm{~cm}^{-1}$ (Fig. 1C) with increasing chain length. Moreover, there is an obvious downshift of the negative ROA band from 1006 to $999 \mathrm{~cm}^{-1}$ with increasing chain length (Fig. 1B).

From the development of formation of these spectra bands we can also derive the minimal proline chain length necessary for creation stable PPII conformation. In the spectra of (L-proline) 12 there are already all characteristic bands visible in poly-L-proline spectra that remain practically identical despite different mean molecular weight of poly-L-proline samples. However, these characteristic bands can be also found in spectrum of (L-proline) 6 (even though with much lower intensity). For that reason, it seems that the proline chain necessary for creation of stable PPII helix consists at least of six proline residues.

\section{Conclusions}

Based on the measurement of Raman and ROA spectra of proline samples with different chain length, we were able to clarify the process of formation of PPII conformation in proline rich peptides and found out the notable spectral ROA bands that mark its presence. Specifically, the negative ROA band at $375 \mathrm{~cm}^{-1}$, positive band at $405 \mathrm{~cm}^{-1}$ and particularly positive band at $945 \mathrm{~cm}^{-1}$ may serve as signs of fully formed PPII helix. Simultaneously, our ROA data indicates that minimal length of (L-proline) ${ }_{N}$ chain allowing the formation of sufficiently stable PPII conformation is $N=6$ in qualitative agreement with VCD study of proline oligopeptides [4].

\section{Acknowledgements}

This work has been supported by the grant Agency of the Czech Republic (Grant No. 202/07/0732) and by the Ministry of Education of the Czech Republic (No. MSM 0021620835). 


\section{References}

[1] L.D. Barron, E.W. Blanch and L. Hecht, Adv. Prot. Chem. 62 (2002), 51.

[2] E.W. Blanch, A.C. Gill, A.G.O. Rhie, J. Hope, L. Hecht, K. Nielsen and L.D. Barron, J. Mol. Biol. 343 (2004), 476.

[3] E.W. Blanch, L. Hecht and L.D. Barron, Methods 29 (2003), 196.

[4] R.K. Dukor and T.A. Keiderling, Biopolymers 31 (1991), 1747.

[5] J. Hanzlíková, P. Praus and V. Baumruk, J. Mol. Struct. 431 (1999), 480.

[6] W.C. Johnson, Methods Biochem. Anal. 31 (1985), 61.

[7] J. Kapitán, V. Baumruk and P. Bouř, J. Am. Chem. Soc. 128 (2006), 1438.

[8] J. Kapitán, V. Baumruk, H. Hulačová and P. Maloň, Vibrational Spectrosc. 42 (2006), 88.

[9] T.A. Keiderling, in: Circular Dichroism and Confromational Analysis of Biomolecules, G.D. Fassman, ed., Plenum Press, New York, 1996, p. 555.

[10] I.H. McColl, E.W. Blanch, A.C. Gill, A.G.O. Rhie, M.A. Ritchie, L. Hecht, K. Nielsen and L.D. Barron, J. Am. Chem. Soc. 125 (2003), 10019.

[11] I.H. McColl, E.W. Blanch, L. Hecht, N.R. Kallenbach and L.D. Barron, J. Am. Chem. Soc. 126 (2004), 5076.

[12] Z. Shi, R.W. Woody and N.R. Kallenbach, Adv. Prot. Chem. 62 (2002), 163. 


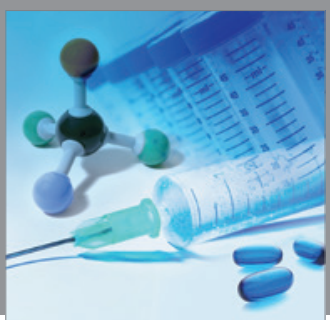

International Journal of

Medicinal Chemistry

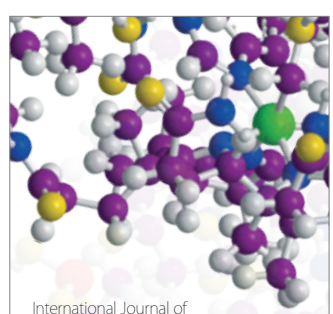

Carbohydrate Chemistry

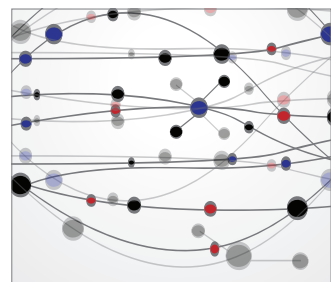

The Scientific World Journal
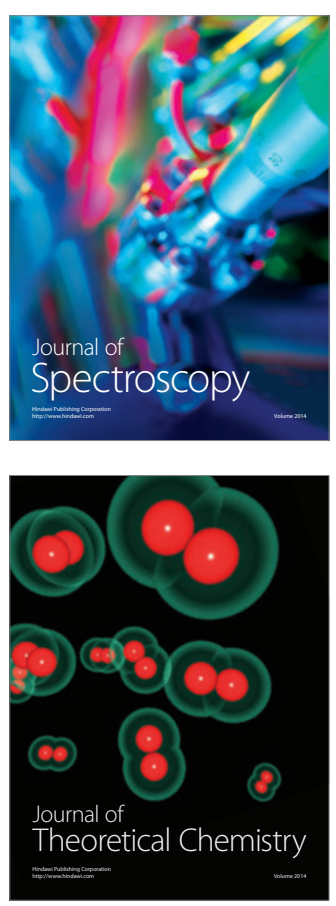
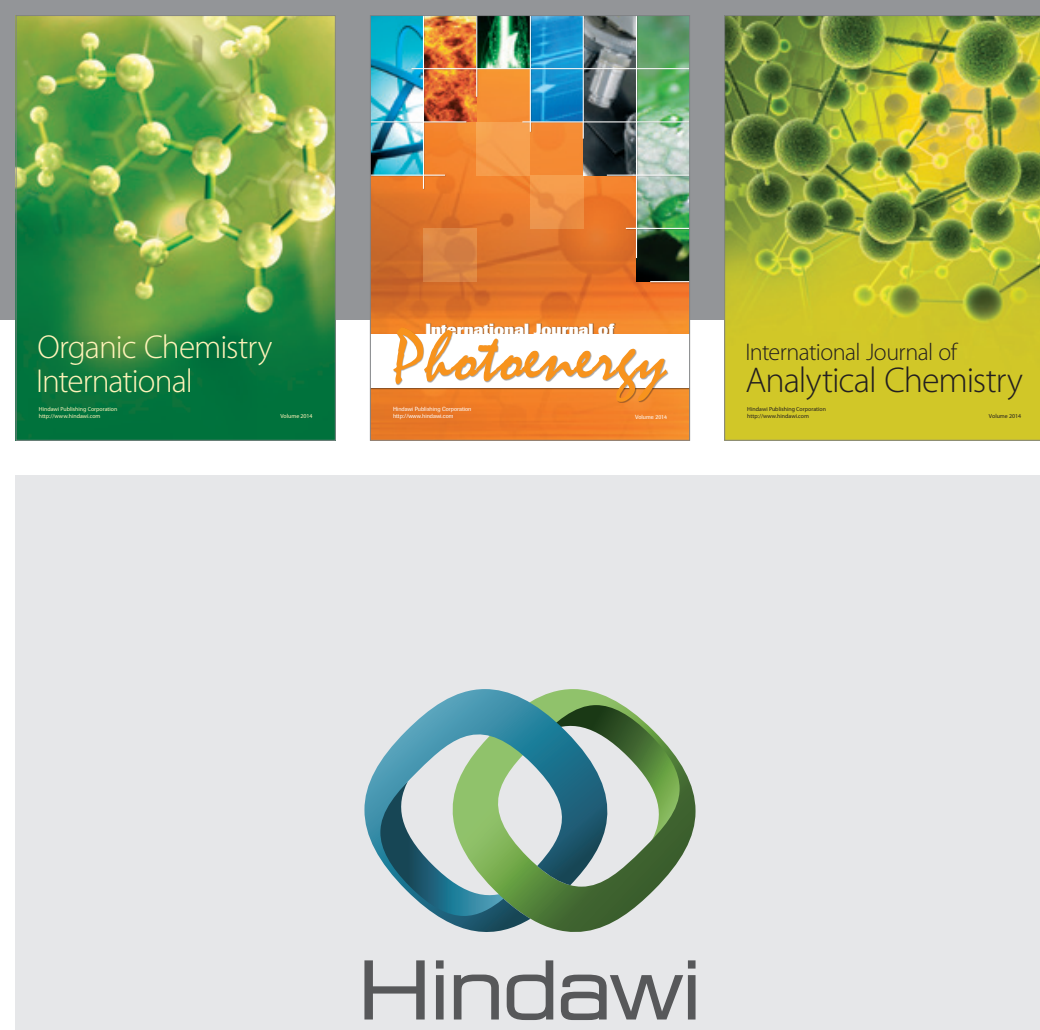

Submit your manuscripts at

http://www.hindawi.com
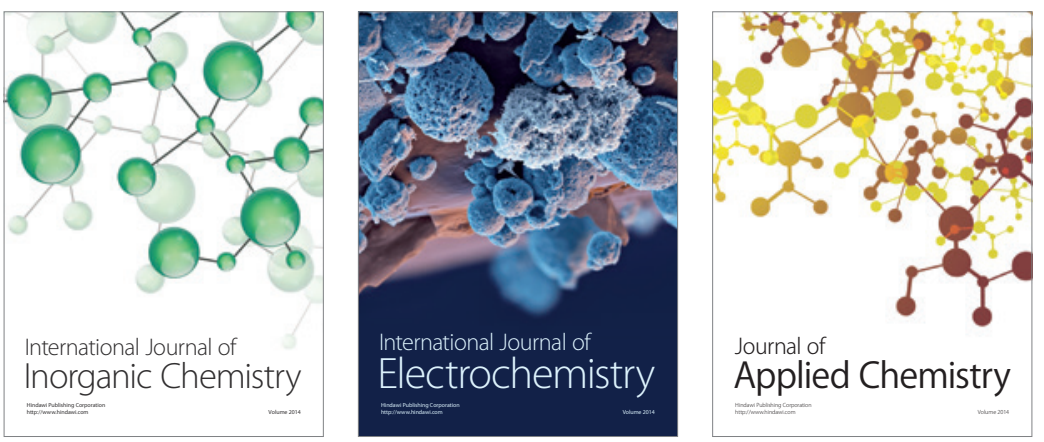

Journal of

Applied Chemistry
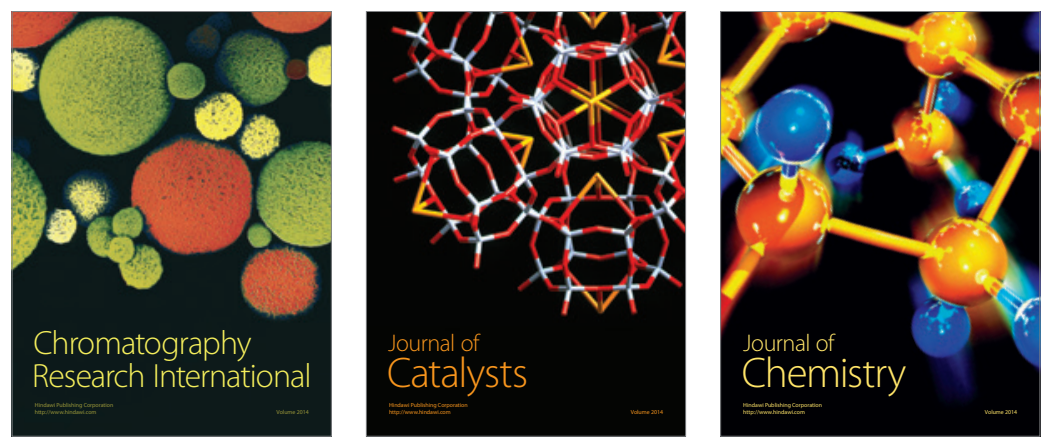
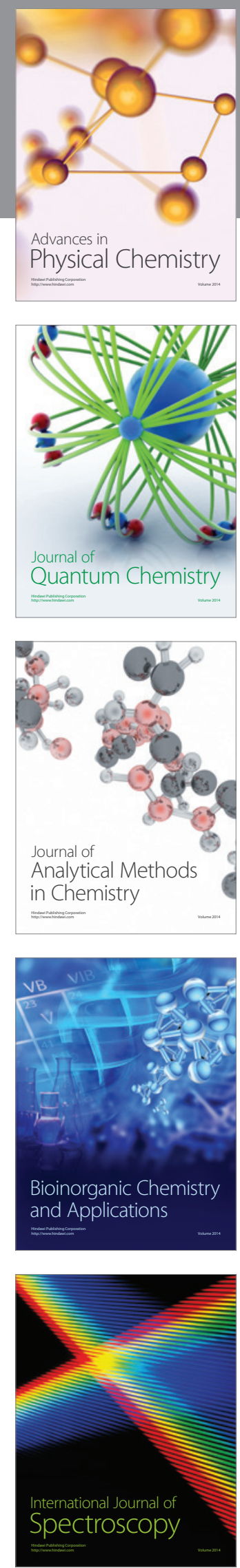